\title{
On the Covering Multiplicity of Lattices
}

\author{
J. H. Conway ${ }^{1}$ and N. J. A. Sloane ${ }^{2}$ \\ ${ }^{1}$ Mathematics Department, Princeton University, \\ Princeton, NJ 08540, USA \\ ${ }^{2}$ Mathematical Sciences Research Center, AT\&T Bell Laboratories, \\ Murray Hill, NJ 07974, USA
}

\begin{abstract}
Let the lattice $\Lambda$ have covering radius $R$, so that closed balls of radius $R$ around the lattice points just cover the space. The covering multiplicity $C M(\Lambda)$ is the maximal number of times the interiors of these balls overlap. We show that the least possible covering multiplicity for an $n$-dimensional lattice is $n$ if $n \leq 8$, and conjecture that it exceeds $n$ in all other cases. We determine the covering multiplicity of the Leech lattice and of the lattices $I_{n}, A_{n}, D_{n}, E_{n}$ and their duals for small values of $n$. Although it appears that $C M\left(I_{n}\right)=2^{n-1}$ if $n \leq 33$, as $n \rightarrow \infty$ we have $C M\left(I_{n}\right) \sim 2.089 \ldots n$. The results have application to numerical integration.
\end{abstract}

\section{Introduction}

A problem of recent interest (cf. Sullivan [18]) is to find the smallest possible covering multiplicity (defined in the Abstract) of any $n$-dimensional lattice, and to calculate the covering multiplicities of various well-known lattices. Figure 1 shows an example. The covering multiplicity so defined is of use in estimating various integrals, since if $I$ is the integral of some function over the whole space and $I_{B}$ its integral over one of the covering balls, then

$$
I \leq \sum_{B} I_{B} \leq C M(\Lambda) I
$$

In this paper we determine the covering multiplicities of certain members of the families $I_{n}(n \geq 1), A_{n}(n \geq 1), D_{n}(n \geq 1), E_{n}(n=6,7,8)$ and their duals $A_{n}^{*}$, $D_{n}^{*}, E_{n}^{*}$ for small values of $n$ (the results are given in Table 1) and of the Leech lattice $\Lambda_{24}$ (for which the covering multiplicity is 25 ). 


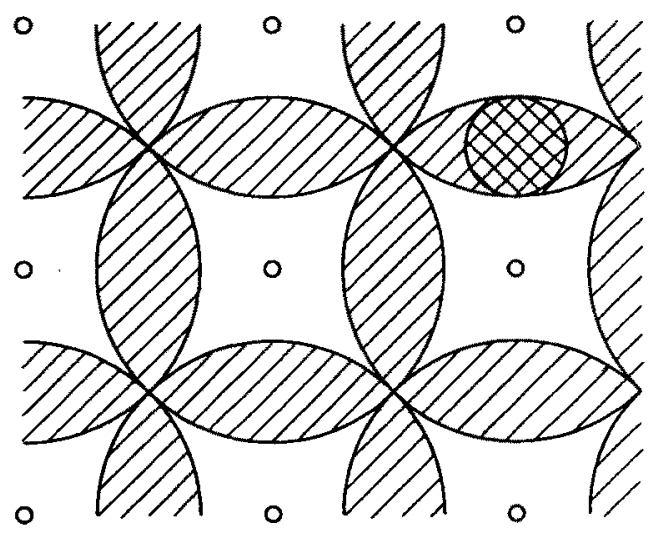

Fig. 1. The covering multiplicity of the square lattice $I_{2}$ (small circles) is 2 , since all points in the open lunes (shaded) are covered twice. An umbral circle is shown doubly shaded.

In particular we will show that $C M\left(A_{n}^{*}\right)=n$ for $n \leq 8$, and also that $C M\left(D_{4}\right)=4$ and $C M\left(E_{6}^{*}\right)=6$. Since the covering multiplicity of an $n$-dimensional lattice is at least $n$ (see Theorem 2), this will establish our main result.

Theorem 1. The minimal covering multiplicity of an $n$-dimensional lattice is $n$ if $n \leq 8$.

We have also made experimental investigations of these lattices in higher dimensions, obtaining lower bounds on their covering multiplicities, some of which are shown in Table 1. (We conjecture that the lower bounds shown in the table are in fact the true values.)

The results in Table 1 (and more extensive experiments not reported here) lead us to conjecture that the minimal covering multiplicity exceeds $n$ in all dimensions above eight.

Since there are usually many ways to perturb a lattice without changing its covering multiplicity, the lattices in Table 1 are of course not the only ones with these values of the covering multiplicity.

Table 1. Covering multiplicities of root lattices and duals.

\begin{tabular}{rrrrrrrr}
\hline$n$ & \multicolumn{1}{c}{$I_{n}$} & \multicolumn{1}{c}{$A_{n}$} & $A_{n}^{*}$ & $D_{n}$ & $D_{n}^{*}$ & $E_{n}$ & $E_{n}^{*}$ \\
\hline 1 & 1 & 1 & 1 & 1 & 1 & & \\
2 & 2 & 2 & 2 & 2 & 2 & & \\
3 & 4 & 4 & 3 & 4 & 3 & & \\
4 & 8 & 7 & 4 & 4 & 4 & & \\
5 & 16 & 15 & 5 & 11 & 6 & & \\
6 & $\geq 32$ & $\geq 21$ & 6 & $\geq 16$ & 8 & 17 & 6 \\
7 & $\geq 64$ & $\geq 56$ & 7 & $\geq 32$ & $\geq 12$ & 28 & 9 \\
8 & $\geq 128$ & $\geq 84$ & 8 & $\geq 64$ & $\geq 16$ & 9 & 9 \\
9 & $\geq 256$ & $\geq 210$ & $\geq 11$ & $\geq 163$ & $\geq 24$ & & \\
\hline
\end{tabular}


It is worth pointing out that there is a simple way to compare lattices that have the same covering multiplicity. This is by finding the largest sphere whose interior is wholly contained in the set of points covered $C M(\Lambda)$ times: we call this the umbral sphere of the lattice. It is illustrated in Fig. 1 for the square lattice $I_{2}$. Let $R_{\mathrm{u}}$ be the radius of the umbral sphere when the lattice is scaled so as to have determinant 1 . We can then ask: among all lattices with the minimal covering multiplicity, which has the smallest value of $R_{\mathrm{u}}$ ? We show how to compute $R_{\mathrm{u}}$ in Section 2-see (4). For example, although both $A_{4}^{*}$ and $D_{4}$ have covering multiplicity 4 , the value of $R_{\mathrm{u}}$ for $A_{4}^{*}$ is

$$
\frac{\sqrt{\frac{2}{5}}-\sqrt{\frac{19}{50}}}{\left(\frac{1}{5}\right)^{1 / 8}}=0.019582 \ldots
$$

while for $D_{4}$ it is

$$
\frac{1-\sqrt{3 / 2}}{4^{1 / 8}}=0.112658 \ldots
$$

Similarly, the values of $R_{\mathrm{u}}$ for $A_{6}^{*}$ and $E_{6}^{*}$ (which both have covering multiplicity 6) are

$$
\frac{2 / \sqrt{7}-\sqrt{\frac{55}{98}}}{\left(\frac{1}{7}\right)^{1 / 12}}=0.007973 \ldots
$$

and

$$
\frac{\sqrt{\frac{2}{3}}-\sqrt{\frac{11}{18}}}{\left(\frac{1}{3}\right)^{1 / 12}}=0.038093 \ldots
$$

respectively.

The $n$-dimensional cubic lattice $I_{n}$ is of particular interest. Our investigations strongly suggest that the sequence of values of $C M\left(I_{n}\right)$ has a rather unusual behavior: $C M\left(I_{n}\right)=2^{n-1}$ for all $n \leq 33$, while $C M\left(I_{n}\right)>2^{n-1}$ for all $n>33$. Our best lower bounds on $C M\left(I_{n}\right)$ are shown in Table 2 . It follows from the work of Mazo and Odlyzko [12] that

$$
C M\left(I_{n}\right) \sim 2.089097 \ldots{ }^{n+o(\sqrt{ } n)}
$$

as $n \rightarrow \infty$. We discuss $I_{n}$ in Section 3. The lattices $A_{n}, A_{n}^{*}, D_{n}, \ldots$ are dealt with in turn in Sections $4,5, \ldots$, ending with the Leech lattice $\Lambda_{24}$ in Section 9.

We remark that, once the covering radius $R$ of a lattice $\Lambda$ has been found, it is trivial to obtain the average covering multiplicity. This is the average number of times a point is in the interior of the covering balls, which is equal to the covering density (or thickness) of $\Lambda$, given by

$$
\frac{V_{n} R^{n}}{\sqrt{\operatorname{det} \Lambda}}
$$


Table 2. Lower bounds to covering multiplicities of cubic lattices $I_{n}(r$ is defined in Section 3$)$.

\begin{tabular}{clc}
\hline$n$ & \multicolumn{1}{c}{$C M\left(I_{n}\right) \geq$} & $r$ \\
\hline $1-33$ & $2^{n-1}$ & $n-1$ \\
34 & $2^{33}+10850970$ & 1 \\
35 & $2^{34}+341772656$ & 2 \\
36 & $2^{35}+1329309096$ & 3 \\
37 & $2^{36}+7307575295$ & 0 \\
38 & $2^{37}+17587471742$ & 1 \\
39 & $2^{38}+41179889288$ & 2 \\
40 & $2^{39}+94490796376$ & 3 \\
\hline
\end{tabular}

where $V_{n}$ is the volume of an $n$-dimensional sphere of radius 1 [3, p. 31]. Finding $C M(\Lambda)$ appears to be a much more difficult problem.

For example, the average covering multiplicities of the lattices $I_{n}, D_{n}$, and $A_{n}^{*}$ are, respectively,

$$
\begin{aligned}
& \left(\sqrt{\frac{\pi e}{2}}\right)^{n+o(n)}=2.066365 \ldots{ }^{n+o(n)}, \\
& \left(\sqrt{\frac{\pi e}{2}}\right)^{n+o(n)}=2.066365 \ldots{ }^{n+o(n),} \\
& \left(\sqrt{\frac{\pi e}{6}}\right)^{n+o(n)}=1.193016 \ldots{ }^{n+o(n)} .
\end{aligned}
$$

We call a point of $\mathbb{R}^{n}$ which is in the interior of $C M(\Lambda)$ balls a thickest point for $\Lambda$.

Note that since only open balls are used in determining $C M(\Lambda)$, points of $\mathbb{R}^{n}$ that are maximally distant from $\Lambda$ (the "deep holes" in $\Lambda$ ) are usually not thickest points. However, we will see several examples later where thickest points are deep holes in sublattices.

This work has involved considerable use of computers.

(1) We searched for thickest points in a lattice by choosing points at random from a fundamental parallelepiped, and computing the number of open balls of radius $R$ that contain them. However, it appears that for many lattices the probability that a random point is a thickest point is very small, so this approach is only successful in low dimensions.

(2) For some lattices we used MACSYMA [11] to determine how many open balls contain a point $P$, by computing the theta series with respect to $P$-see Sections 3 and 6.

(3) It is often necessary to determine if the open balls of radius $R$ about lattice points $P_{1}, \ldots, P_{M} \in \Lambda$ have a common point. This can be answered by computing the radius $r$ of the smallest sphere that contains $P_{1}, \ldots, P_{M}$. (There is a common point if and only if $r<R$.) 
Although this smallest containing sphere problem has been studied by several authors [6]-[8], [13], [16, p. 248], [17], there does not seem to be a standard name for the containing sphere. We call it the perisphere, ${ }^{1}$ and similarly refer to the pericenter and periradius of $P_{1}, \ldots, P_{M}$. The square of the periradius is the perinorm of $P_{1}, \ldots, P_{M}$.

Smallest containing sphere problems can be solved very efficiently using the AMPL [9] and MINOS [15] programs. ${ }^{2}$

(4) We also frequently use AMPL and MINOS to compute the distance from a given point $P$ to a convex polytope $\Pi$ (usually a fundamental simplex for the lattice in question - see, for example, the proof of Theorem 6). ${ }^{3}$

Since we make heavy use of the results from AMPL and MINOS in proving some of our theorems, it is appropriate to discuss the accuracy and rigor of these procedures.

(a) AMPL [9] is simply a flexible front-end, which transforms the problem into the appropriate format for MINOS or other optimization programs.

(b) MINOS [15] was developed to handle much larger problems than ours, which typically involve minimizing a function of eight variables subject to eight constraints. Running times on our problems were around 0.20 seconds, and the results were correct to at least seven significant figures.

(c) Our problems - see footnotes 2 and 3 -involve minimizing a quadratic function, usually of the form $x_{1}^{2}+\cdots+x_{n}^{2}$, subject to linear constraints. MINOS solves such problems using a reduced-gradient algorithm, following Murtagh and Saunders [14]. Since our problems are so small, any particular solution found by MINOS could be verified by hand. The total number of calls to MINOS used in all our proofs was less than 10,000 . So although some of these proofs depend on computers, the same results could in principal be obtained without their help.

We assume the reader is familiar with the lattices mentioned (any other course would have doubled the length of the paper) although we do give definitions for the principal lattices. For further information see [1], [2], [5], [10], and especially [3].

The subscript on the name of a lattice gives its dimension, and an asterisk indicates the dual lattice. We note that $I_{n}^{*}=I_{n}, E_{8}^{*}=E_{8}$, and $\Lambda_{24}^{*}=\Lambda_{24}$. Also $A_{1} \cong A_{1}^{*} \cong D_{1} \cong D_{1}^{*} \cong I_{1}, A_{2}^{*} \cong A_{2}, D_{2} \cong I_{2}, D_{3} \cong A_{3}$, and $D_{4}^{*} \cong D_{4}$, where $\cong$ indicates geometrically similar lattices.

The cell structures (i.e., the Voronoi and Delaunay polytopes) of the root lattices

\footnotetext{
1 The prefix "peri" means "around."

${ }^{2}$ An appropriate formulation of such a problem for AMPL and MINOS is the following. Let $C$ be the pericenter and let $s$ be the perinorm. Then set $t=s-C \cdot C$. and call AMPL and MINOS to minimize $t+C \cdot C$ subject to $t \geq P_{i} \cdot P_{i}-2 C \cdot P_{i}, i=1, \ldots, M$. We thank Steve Fortune for this observation.

${ }^{3}$ Let $\Pi$ be defined by the inequalities $\pi_{i} \cdot z \geq a_{i}, i=1, \ldots, M$. Let $z$ be the closest point of $\Pi$ to $P$, and set $y=P-z$. Then we call AMPL and MINOS to minimize $y \cdot y$ subject to $\pi_{i} \cdot y \leq \pi_{i} \cdot P-a_{i}$, $i=1, \ldots, M$.
} 
$I_{n}, A_{n}, D_{n}, E_{n}$ and their duals are described in [3], [5], [19], and [20]. The reader is particularly referred to [4], where all these cell structures are obtained in a systematic and uniform manner.

The norm or squared length $x \cdot x$ of a vector $x$ is denoted throughout by $N(x)$.

\section{General Results}

We first prove that the covering multiplicity of an $n$-dimensional lattice is at least $n$. This is a consequence of the following more general result.

Theorem 2. Let $\Sigma$ be a set of points in $\mathbb{R}^{n}$ for which there are positive numbers $a$, $R$ such that dist $(s, t) \geq a$ for all $s, t \in \Sigma, s \neq t$, and such that the closed balls of radius $R$ about the points of $\Sigma$ cover $\mathbb{R}^{n}$. Then there is a point $w \in \mathbb{R}^{n}$ in the interior of at least $n$ of these balls.

For the proof we need a lemma.

Lemma 3. Suppose $v_{1}, \ldots, v_{M}(M \geq n)$ are vectors in $\mathbb{R}^{n}$ such that any nonzero vector has positive inner product with at least one $v_{i}$. Then there is a vector $w \in \mathbb{R}^{n}$ with positive inner product with at least $n$ of the $v_{i}$.

Proof. The proof is by induction on $n$. Choose one of the $v_{i}$ 's, $v$ say, and let $\Pi$ be the $(n-1)$-dimensional subspace orthogonal to $v$. Each vector in $\Pi$ has positive inner product with at least one of the other $v_{i}$ 's, and so by the inductive hypothesis there is a vector $x \in \Pi$ with positive inner product with at least $n-1$ of them, say $x \cdot v_{i}=b_{i}>0,1 \leq i \leq n-1$. Choose $\varepsilon$ to satisfy $0<\varepsilon<b_{i} /\left|v \cdot v_{i}\right| \neq 0$. Then $w=x+\varepsilon v$ is a vector with the desired properties.

Proof of Theorem 2. Let $R^{\prime} \leq R$ be the smallest number such that closed balls of radius $R^{\prime}$ around the points of $\Sigma$ cover $\mathbb{R}^{n}$, and let $P$ be a critical point for the covering, i.e., a point which would not be covered if the balls were shrunk further. We take $P$ as the origin, and let $v_{1}, \ldots, v_{M}$ be the points in $\Sigma$ at distance $R^{\prime}$ from $P$. The $v_{i}$ satisfy the hypothesis of Lemma 3 , and the point $\varepsilon w$, for sufficiently small $\varepsilon>0$, is in the interior of at least $n$ of the balls.

We now show that the behavior illustrated in Fig. 1 is typical of all lattices, in the sense that the regions inside sets of $C M(\Lambda)$ intersecting balls are always disjoint from each other (just as the open lunes in Fig. 1 are disjoint).

Theorem 4. For each set $\mathscr{S}$ of $C M(\Lambda)$ lattice points for which the corresponding balls have a common intersection, let $U(\mathscr{S})$ denote their open intersection. Then the regions $U(\mathscr{S})$ are disjoint.

Proof. A point $P$ of the boundary of $U(\mathscr{S})$ is not in the interior of any ball with center $x \notin \mathscr{S}$, or else some point near $P$ would be contained in more than $C M(\Lambda)$ 
balls. On the other hand, $P$ is not in the interior of at least one of the original balls. Therefore points on the boundary of $U(\mathscr{S})$ are not covered $C M(\Lambda)$ times.

Theorem 4 implies that we can compute the umbral spheres for $\Lambda$ (defined in Section 1) as follows. Let $\rho$ be the smallest periradius of any such set $\mathscr{S}$. Then the umbral spheres have radius $R-\rho$, where $R$ is the covering radius of the lattice, and so

$$
R_{\mathrm{u}}=\frac{R-\rho}{(\operatorname{det} \Lambda)^{1 /(2 n)}}
$$

For many of the lattices we consider (although not for $E_{7}$ or $\Lambda_{24}$ ), all sets $\mathscr{S}$ have the same periradius.

\section{The $\boldsymbol{n}$-Dimensional Cubic Lattice $\boldsymbol{I}_{\boldsymbol{n}}$}

$I_{n}$ consists of the vectors $x_{1} x_{2} \cdots x_{n}$ with all $x_{i} \in \mathbb{Z}$, and has covering radius $R=\sqrt{n} / 2$ (see Section 5 of Chapter 4 in [3]). Clearly, $C M\left(I_{1}\right)=1$, so we may assume $n \geq 2$. The point $0 \frac{1}{2} \frac{1}{2} \cdots \frac{1}{2}$ is in the interior of the balls of radius $R$ centered at all lattice points $0 * * \cdots *$, where $*$ indicates a coordinate that is 0 or 1 , and so

$$
C M\left(I_{n}\right) \geq 2^{m-1} .
$$

The numerical evidence in Table 2 suggests that equality may hold in (5) if and only if $n \leq 33$. However, we can establish the equality only for smaller values of $n$.

\section{Theorem 5.}

$$
C M\left(I_{n}\right)=2^{n-1} \quad \text { for } n \leq 5 .
$$

Proof. We give the proof when $n=5$, the other cases being similar and easier. We may assume that a thickest point has the form $P=v$ w $x$ y $z$, where $0 \leq v \leq w \leq x \leq y \leq z \leq \frac{1}{2}$. It is easy to check by hand that only 37 lattice points are within norm $R^{2}=\frac{5}{4}$ of such a point, namely the 32 points $* * * * *$ (where $*=0$ or 1$)$ and the five points $(-10000)$, where the parentheses indicate that all cyclic shifts of the parenthesized coordinates are to be included. We must show that no subset $\mathscr{S}$ of 17 of these 37 points has perinorm $<\frac{5}{4}$.

A basic principle which we use repeatedly is that a set of balls of radius $R$ with a common interior point cannot include two balls with centers $A$ and $B$ satisfying

$$
N(A-B) \geq 4 R^{2} .
$$

(For the interiors of two such balls are disjoint.) 
Case 1: $\mathscr{S}$ contains -10000 and $0-1000$. By the above principle the other points in $\mathscr{S}$ can only include the ten points 10000,01000 and $00 * * *$, and so $|\mathscr{S}| \leq 12$.

Case 2: $\mathscr{S}$ contains no vector with negative components. In this case there are 32 candidates for $\mathscr{P}$, the points $* * * * *$. We form what we call the exclusion graph for these points: there is a node for each point, and two nodes are joined by an edge if and only if the corresponding points $A$ and $B$ satisfy (7). Then $|\mathscr{P}|$ clearly cannot exceed the independence number of this graph (the maximal size of an independent set of nodes).

In the present example the graph consists of 16 disjoint edges, and so $|\mathscr{F}| \leq 16$.

Case 3: $\mathscr{S}$ contains a single vector with a negative coordinate, say -100000. The only other vectors that can occur in $\mathscr{S}$ are the 17 vectors 10000 and $0 * * * *$, and we cannot have both 10000 and $0 * * * *$. If 100000 does not occur we are back to Case 2 . Otherwise $\mathscr{S}$ consists of the 17 vectors -10000 and $0 * * * *$, whose perinorm (found by AMPL and MINOS, see footnote 2) is $\frac{13}{9}>\frac{5}{4}$. Thus no 17 -subset $\mathscr{S}$ exists, and so $C M\left(I_{5}\right)=16$.

To obtain better lower bounds than (6) for larger values of $n$ we make use of theta series. Points of the form $P=0^{n-r} \frac{1 r}{2}$ for $0 \leq r \leq n-1$ appear to be good candidates for thickest points in $I_{n}$. The theta series of $I_{n}$ with respect to $P$ is $[3$, p. 106]

$$
\begin{aligned}
\sum_{x \in I_{n}} q^{N(x-P)} & =\theta_{2}(q)^{r} \theta_{3}(q)^{n-r} \\
& =\sum A_{1} q^{t} \quad \text { (say), }
\end{aligned}
$$

where

$$
\theta_{2}(q)=\sum_{m=-\infty}^{\infty} q^{(m+1 / 2)^{2}}, \quad \theta_{3}(q)=\sum_{m=-\infty}^{\infty} q^{m^{2}}
$$

and $A_{t}$ denotes the number of lattice points at squared distance $t$ from $P$.

We use MACSYMA [11] to calculate $\sum_{t<R} A_{t}$ (the number of balls containing $P$ ) and to maximize this over $r$. The results for $n \leq 40$, together with a best choice for $r$, are shown in Table 2.

It follows from the work of Mazo and Odlyzko [12] that, as $n \rightarrow \infty$,

$$
C M\left(I_{n}\right) \sim 2.089097 \ldots{ }^{n+O(\sqrt{ })} .
$$

This is the case $\alpha=\frac{1}{4}$ in [12]; the particular constant needed for (9) is not given in Table 1 of [12] but was kindly supplied by Andrew Odlyzko. In [12] it is also shown that any point of the form $0^{n-r} \frac{1 r}{2}$ for fixed $r$ is in $c^{n+o(n)}$ balls as $n \rightarrow \infty$, where $c$ is strictly less than the constant in (9). 


\section{The Lattice $\boldsymbol{A}_{\boldsymbol{n}}$}

$A_{n}$ consists of those points $x_{0} x_{1} \cdots x_{n} \in I_{n+1}$ satisfying $\sum x_{i}=0$, and has squared covering radius $R^{2}=a(n+1-a) /(n+1)$, where $a=[(n+1) / 2]$ (see Section 6.1 of Chapter 4 in [3]). It is trivial to see that $C M\left(A_{1}\right)=1, C M\left(A_{2}\right)=2$; and the face-centered cubic lattice $A_{3} \cong D_{3}$ is discussed in Section 6 .

For $A_{2 t}$ the point

$$
\left(\frac{t+2}{2 t+1}\right)^{t-1}\left(\frac{-t+1}{2 t+1}\right)^{t+2}
$$

is certainly contained in the balls centered at points of the form

$$
x_{1} \cdots x_{t-1} ;-x_{1} \cdots-x_{t-1} 0 \cdots 0,
$$

$x_{i}=0$ or 1 (and in other balls when $n$ is large), so that

$$
C M\left(A_{2 t}\right) \geq \sum_{i=0}^{t-1}\left(\begin{array}{c}
t-1 \\
i
\end{array}\right)\left(\begin{array}{c}
t+2 \\
i
\end{array}\right)=\left(\begin{array}{c}
2 t+1 \\
t-1
\end{array}\right) .
$$

Similarly the point

$$
\left(\frac{t+2}{2 t+2}\right)^{t}\left(\frac{-t}{2 t+2}\right)^{t+2}
$$

shows that

$$
C M\left(A_{2 \mathfrak{t}+1}\right) \geq \sum_{i=0}^{t}\left(\begin{array}{l}
t \\
i
\end{array}\right)\left(\begin{array}{c}
t+2 \\
i
\end{array}\right)=\left(\begin{array}{c}
2 t+2 \\
t
\end{array}\right)
$$

Equations (10) and (11) justify the lower bounds in Table 1 for $n \geq 5$. When $n=4,(10)$ can be strengthened, since the point $\left(\frac{3}{7}\right)^{2}\left(-\frac{2}{7}\right)^{3}$ is contained in seven spheres.

Theorem 6. The covering multiplicities of $A_{1}, \ldots, A_{5}$ are respectively $1,2,4,7,15$.

Proof. We begin with the proof for $A_{4}$; this is the prototype of many later proofs.

A fundamental simplex for the (infinite) affine Weyl group of type $A_{n}$ is defined by the inequalities

$$
x_{0} \leq x_{1}, \quad x_{1} \leq x_{2}, \quad \ldots, \quad x_{n-1} \leq x_{n}, \quad x_{n} \leq x_{0}+1
$$

(see [1], [5], and especially Chapter 21, Fig. 21.1, of [3]). We consider a thickest point $P$ for $A_{4}$ contained in the fundamental simplex. In particular, $P$ is in the ball centered at the origin. The center of any other ball containing $P$ is at squared 


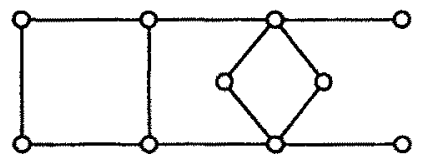

0

Fig. 2. Exclusion graph for $A_{4}$.

distance $<4 R^{2}=\frac{24}{5}=4.8$ from the origin. Since the theta series of $A_{4}$ (with respect to the origin) is

$$
1+20 q^{2}+30 q^{4}+\cdots
$$

there are 51 possible balls to consider.

We now use AMPL and MINOS (see footnote 3) to compute the squared distance from each of these points to the fundamental simplex. For only 12 of the 51 points is this less than $R^{2}=\frac{6}{5}$. The exclusion graph of these 12 points (defined in the previous section) is shown in Fig. 2. It is easily seen (by eye) that the independence number of this graph is 8 , and that there are exactly two independent sets of size 8. However (from AMPL and MINOS), the perinorm of each such set is exactly $\frac{6}{5}$. Thus only seven open balls can intersect, and $C M\left(A_{4}\right)=7$.

The argument for $A_{5}$ is similar. Now $R^{2}=\frac{3}{2}, 4 R^{2}=6$, and the theta series is $1+30 q^{2}+90 q^{4}+140 q^{6}+\cdots$, so there are 261 vectors to be considered. Only 27 of them are at distance $<R$ from the fundamental simplex, and we form the 27-node exclusion graph, in which we must show that there is no maximal independent set of size $\geq 16$. There are five nodes with too high a degree to be part of such a set, and (after their removal) six isolated nodes which must be part of such a set. However, the remaining 16-node graph contains eight disjoint edges.

\section{The Lattice $A_{n}^{*}$}

The dual lattice $A_{n}^{*}$ is the union of $n+1$ cosets $[i]+A_{n}$ of $A_{n}$, where

$$
[i]=\left(\frac{-i}{n+1}\right)^{j}\left(\frac{j}{n+1}\right)^{i},
$$

and $i+j=n+1,0 \leq i \leq n$. The Delaunay cells are copies of the fundamental simplex for $A_{n}$, for instance that with vertices [i], $0 \leq i \leq n$. (This simplex is the same as that defined in (12).) The covering radius $R$ of $A_{n}^{*}$ is given by

$$
R^{2}=\frac{n(n+2)}{12(n+1)}
$$

(For further information about this lattice see [3, Chapter 4, Section 6.6] and [4].) 


\section{Theorem 7.}

$$
C M\left(A_{n}^{*}\right)=n \quad \text { for } n \leq 8
$$

Proof. By Theorem 2, $C M\left(A_{n}^{*}\right) \geq n$. We may assume that a thickest point $P$ is in the fundamental simplex with vertices $[i], 0 \leq i \leq n$. Let $\mathscr{S}$ be the set of centers of a collection of $C M\left(A_{n}^{*}\right)$ open balls containing $P$. For $n \leq 5$ we have $4 R^{2}<2$, and so $\mathscr{S}$ cannot contain two points from the same coset $[i]+A_{n}$, implying $|\mathscr{S}| \leq n+1$. (This is because if $u, v \in[i]+A_{n}, u \neq v$, then $u-v \in A_{n}$ and so $N(u-v) \geq 2$.) Furthermore (still for $n \leq 5$ ) we cannot have $|\mathscr{P}|=n+1$, for the $n+1$ closed balls centered at the vertices [i], $0 \leq i \leq n$, meet at the center of the simplex but do not have a common interior point. Thus

$$
C M\left(A_{n}^{*}\right) \leq n \quad \text { for } n \leq 5 \text {. }
$$

We now discuss the case $n=8$, omitting $n=6$ and 7 which are similar and easier.

Since $R^{2}=\frac{20}{27}, 4 R^{2}=\frac{80}{27}$, and the theta series of $A_{8}^{*}$ begins

$$
1+18 q^{8 / 9}+72 q^{14 / 9}+240 q^{2}+252 q^{20 / 9}+504 q^{26 / 9}+\cdots,
$$

we must consider a population $\Omega$ of $1+18+\cdots+504=1087$ lattice vectors. These are distributed among the nine cosets as shown in Table 3.

We must show that no 9 -subset $\mathscr{S}$ of $\Omega$ corresponds to a set of intersecting spheres. There cannot be just one vector from each coset, by the argument at the end of the proof for $n=5$, so there must be $\geq 2$ vectors from some coset. We may also assume if we wish that $0 \in \mathscr{S}$. We proceed to establish several properties of $\mathscr{S}$.

(i) $\mathscr{S}$ does not contain four vectors $u, v, u, x$ from the same coset.

Proof. We may take $u=0$. Then $v, w, x$ and their differences must have norm 2 , say $v=1-10^{7}, w=10-10^{6}, x=10^{2}-10^{5}$. But the perinorm of this set is $\frac{3}{4}>\frac{20}{27}$.

Table 3. Vectors of norm $<\frac{80}{2}$ in $A_{8}^{*}$.

\begin{tabular}{ccccc}
\hline Coset & Norm & Number & Norm & Number \\
\hline 0 & 0 & 1 & 2 & 72 \\
1 & $\frac{8}{9}$ & 9 & $\frac{26}{9}$ & 252 \\
2 & $\frac{14}{9}$ & 36 & - & - \\
3 & 2 & 84 & - & - \\
4 & $\frac{20}{9}$ & 126 & - & - \\
5 & $\frac{20}{9}$ & 126 & - & - \\
6 & 2 & 84 & - & - \\
7 & $\frac{14}{9}$ & 36 & - & - \\
8 & $\frac{8}{9}$ & 9 & $\frac{26}{9}$ & 252 \\
\hline
\end{tabular}


(ii) If $0 \in \mathscr{S}$, then $\mathscr{S}$ does not contain a vector of norm $\frac{26}{9}$.

Proof. We take the particular norm $\frac{26}{9}$ vector

$$
u=-\frac{10}{9}\left(\frac{8}{9}\right)^{2}\left(-\frac{1}{9}\right)^{6}
$$

and examine every triple $0, u, v$, where $v \in \Omega, v \neq 0, v \neq u$. There are only six choices for $v$ for which the perinorm of the triple is $<\frac{20}{27}$. So if $u \in \mathscr{S},|\mathscr{P}| \leq 8$.

(iii) If $\mathscr{S}$ contains two vectors from coset [i] $+A_{8}$, there is at most one vector from $[i+1]+A_{8}$ and at most one from $[i-1]+A_{8}$.

Proof. Suppose $u=0^{9}, v=10^{7}-1 \in \mathscr{S}$. Then the only compatible vector from $[1]+A_{8}$ is $\frac{8}{9}\left(-\frac{1}{9}\right)^{8}$.

(iv) $\mathscr{S}$ does not contain vectors $u, v, w$ with $u-v \in A_{8}, v-w \in A_{8}$, and $N(u-v)=N(v-w)=N(w-u)=2$.

Proof. We may take $u=0^{9}, v=1-10^{7}, w=10-10^{6}$. There are ten possible vectors $x \in \Omega$ such that the perinorm of $u, v, w, x$ is $<\frac{20}{27}$. By use of (i) and (ii) these ten are reduced to five, and so $|\mathscr{P}| \leq 8$.

(v) $\mathscr{S}$ does not contain three vectors from the same coset.

This follows from (ii) and (iv).

(vi) If $\mathscr{S}$ contains two vectors from coset $[i]+A_{8}$, then it contains no vectors from $[j]+A_{8}$ if $|i-j| \equiv 4(\bmod 9)$.

Proof. We may assume $\mathscr{P}$ contains

$$
u=0^{9}, v=10^{7}-1, w=\left(\frac{5}{9}\right)^{4}\left(-\frac{4}{9}\right)^{5} \in[4]+A_{8} .
$$

There are 12 vectors $x$ that can be adjoined, but none belong to cosets [4], [5], or [6]. Therefore $\mathscr{S}$ must contain two vectors $x, x^{\prime}$ from one of cosets [2], [3], or [7]. However, none of the resulting sets $u, v, w, x, x^{\prime}$ can be extended by a sixth vector $x^{\prime \prime}$.

It is now easy to see that there is no way to distribute nine points among the nine cosets while satisfying (iii), (v), and (vi).

The perinorm of the points $[1], \ldots,[n]$ is

$$
\rho^{2}=\frac{(n-1)\left(n^{2}+4 n+6\right)}{12(n+1)^{2}}
$$

This determines the radius of the umbral sphere for $A_{n}^{*}, n \leq 8$ (see Section 1 and (4)).

For larger values of $n$ we have $C M\left(A_{9}^{*}\right) \geq 11$ (by considering the point $\frac{1}{10}^{5}$ $-\frac{1}{10}$ ), $C M\left(A_{10}^{*}\right) \geq 12$ (from $0^{5} \frac{5}{6}-\frac{15}{6}$ ), $C M\left(A_{n}^{*}\right) \geq 2 n+3$ for $n \geq 11$ (from $0^{n+1}$ ), and $C M\left(A_{14}^{*}\right) \geq 34$ (from $\frac{9}{14} 0-\frac{5}{14}{ }^{9}$ ). We do not know the asymptotic behavior of $C M\left(A_{n}^{*}\right)$, although (3) gives a lower bound. 


\section{The Lattice $D_{n}$}

$D_{n}$ consists of those points $x_{1} \cdots x_{n} \in I_{n}$ for which $x_{1}+\cdots+x_{n}$ is even. Since $D_{1} \cong I_{1}, D_{2} \cong I_{2}$ we assume $n \geq 3$. The covering radius $R$ is 1 (if $n \leq 4$ ) or $\sqrt{n} / 2$ (if $n \geq 4$ ). (For further information about $D_{n}$ see Section 7.1 of Chapter 4 in [3].)

Points of the form $P=0^{n-r} a^{r}$, where $a=\frac{1}{2}-\varepsilon, \varepsilon>0$ is small, and $0 \leq r \leq n$, appear to be the best candidates for thickest points. When $r=1$ this yields

$$
C M\left(D_{n}\right) \geq 2^{n-2} \quad(n \geq 3),
$$

although if $n \equiv 1(\bmod 4)$ we get a slightly better result by taking $r=0$, namely

$$
C M\left(D_{4 t+1}\right) \geq 1+\left(\begin{array}{c}
4 t+1 \\
2
\end{array}\right)+\left(\begin{array}{c}
4 t+1 \\
4
\end{array}\right)+\cdots+\left(\begin{array}{c}
4 t+1 \\
2 t
\end{array}\right)
$$

When $n$ is large these lower bounds fail to count all the balls containing $P$, and (just as for $I_{n}$ ) we get better bounds by using the theta series of the lattice with respect to $P$ (compare (8)). For $D_{n}$ this can be shown to be equal to

$$
\frac{1}{2}\left\{\psi_{3}(q)^{r} \theta_{3}(q)^{n-4}+\psi_{4}(q)^{r} \theta_{4}(q)^{n-r}\right\}
$$

where

$$
\begin{aligned}
& \psi_{3}(q)=\sum_{m=-\infty}^{\infty} q^{(m-a)^{2}}, \quad \psi_{4}(q)=\sum_{m=-\infty}^{\infty}(-1)^{m} q^{(m-a)^{2}} \\
& \theta_{4}(q)=\sum_{m=-\infty}^{\infty}(-1) q^{m^{2}}
\end{aligned}
$$

Again we use MACSYMA to determine the number of balls containing $P$ and to optimize over $r$. We have computed this bound for $n \leq 40$ and show the values for $n \leq 20$ in Table 4. (The pattern in the third column breaks down for $n \geq 25$.)

We suspect that many of the lower bounds in Table 4 are exact, although we can only prove this for $n \leq 5$.

Theorem 8. The covering multiplicities of $D_{3}, D_{4}$, and $D_{5}$ are respectively 4,4 , and 11.

Proof. The face-centered cubic lattice $D_{3}$ has two kinds of Delaunay polyhedra, tetrahedra and octahedra, and $R=1$. We can now check that the ball centered at any vertex of a tetrahedral or octahedral Delaunay polyhedron intersects only those faces of the polyhedron that involve that vertex - we omit the details. It follows that points in the Delaunay octahedra are covered at most three times and points in the Delaunay tetrahedra at most four times, so that $C M\left(D_{3}\right)=4$. 
Table 4. Lower bounds to covering multiplicities of the lattices $D_{n}$.

\begin{tabular}{rlr}
\hline$n$ & $C M\left(D_{n}\right) \geq$ & $r$ \\
\hline 3 & $2+2$ & 3 \\
4 & $2^{2}$ & 3 \\
5 & $2^{3}+3$ & 5 \\
6 & $2^{4}$ & 5 \\
7 & $2^{5}$ & 6 \\
8 & $2^{6}$ & 7 \\
9 & $2^{7}+35$ & 9 \\
10 & $2^{8}$ & 9 \\
11 & $2^{9}$ & 10 \\
12 & $2^{10}$ & 11 \\
13 & $2^{11}+462$ & 13 \\
14 & $2^{12}$ & 13 \\
15 & $2^{13}$ & 14 \\
16 & $2^{14}$ & 15 \\
17 & $2^{15}+6435$ & 17 \\
18 & $2^{16}$ & 17 \\
19 & $2^{17}$ & 18 \\
20 & $2^{18}$ & 19 \\
\hline
\end{tabular}

For $D_{4}$ and $D_{5}$ we assume that a thickest point belongs to the fundamental simplex defined by

$$
0 \leq x_{1}+x_{2}, \quad x_{1} \leq x_{2}, \quad x_{2} \leq x_{3}, \quad \ldots, \quad x_{n-1} \leq x_{n}, \quad x_{n-1}+x_{n} \leq 1
$$

[3, Chapter 21, Fig. 21.2]. The proofs now follow those for $A_{4}$ and $A_{5}$.

In the case of $D_{4}$ the exclusion graph consists of a triangle and three isolated vertices, and so $C M\left(D_{4}\right) \leq 4$. Each Delaunay and polytope for $D_{4}$ is an 8-vertex orthoplex (also called a cross-polytope or generalized octahedron, see [4] and [5]). There is a unique way for four balls to intersect: they must be centered at the vertices of a tetrahedral face of an orthoplex cell (for example, the four vertices $(1,0,0,0)$, with pericenter $\frac{1}{4} \frac{1}{4} \frac{1}{4} \frac{1}{4}$ and perinorm $\frac{3}{4}$ ).

In the case of $D_{5}$ the exclusion graph has 20 nodes and contains precisely two maximal independent sets of size 12 , both of which have perinorm $\frac{5}{4}$.

Asymptotically it seems very plausible (although we have not tried to carry this out) that the arguments of [12] can be modified to show that (9) also holds for $D_{n}$.

\section{The Lattice $D_{n}^{*}$}

For the dual lattice $D_{n}^{*}$ see [3, Chapter 4, Section 7.4] and [4]. Since $D_{3}^{*} \cong A_{3}^{*}$ and $D_{4}^{*} \cong D_{4}$ we assume $n \geq 5$. Consideration of the points

$$
\left(\frac{1}{2}-\varepsilon\right)^{t-1} \frac{12}{4} 0^{t-1}
$$




$$
\begin{gathered}
\left(\frac{1}{2}-\varepsilon\right)^{t}\left(\frac{1}{4}+\varepsilon\right)^{1} 0^{t} \\
\left(\frac{1}{2}\right)^{2 n}\left(\frac{1}{4}-\varepsilon\right)^{1} \varepsilon 0^{2 u-1}
\end{gathered}
$$

shows that

$$
\begin{gathered}
C M\left(D_{2 t}^{*}\right) \geq 2 t \\
C M\left(D_{2 t+1}^{*}\right) \geq \sum_{i=0}^{[(t-1) / 2]}\left(\begin{array}{l}
t \\
i
\end{array}\right)+2^{t} \quad(t \text { odd }) \\
C M\left(D_{4 u+1}^{*}\right) \geq 3 \cdot 2^{2 u-1}
\end{gathered}
$$

respectively, and yields the lower bounds in Table 1 .

\section{Theorem 9.}

$$
C M\left(D_{5}^{*}\right)=6, \quad C M\left(D_{6}^{*}\right)=8
$$

Proof. The method used to prove $C M\left(A_{4}\right)=7$ works very easily here. The exclusion graphs for $D_{5}^{*}$ and $D_{6}^{*}$ contain respectively 8 and 12 nodes. In both cases these nodes are subsets of the vertices of the Delaunay polytope (see [4]). In the case of $D_{6}^{*}$, for example, the Delaunay polytope is the 16-vertex cross-join of two three-dimensional cubes in orthogonal spaces. There is a unique arrangement of eight intersecting balls, whose centers are obtained by taking four nonopposite vertices out of the eight vertices of each of the two cubes.

\section{The Lattices $E_{n}$ and $E_{n}^{*}(n=6,7,8)$}

$E_{8}$ is the union of $D_{8}$ and the translate $\frac{1}{2} \frac{1}{2} \frac{1}{2} \frac{1}{2} \frac{1}{2} \frac{1}{2} \frac{1}{2} \frac{1}{2}+D_{8}$. The covering radius $R$ is 1 , and there are two kinds of Delaunay polytopes, orthoplexes centered at deep holes such as $10^{7}$ and simplexes centered at shallow holes such as $\frac{17}{3}-\frac{1}{3}$ (see [3, Chapter 4, Section 8.1], [4], and [5]).

\section{Theorem 10.}

$$
C M\left(E_{8}\right)=9
$$

Proof. The point $\frac{1}{3}^{7}-\frac{1}{3}$ is contained in the balls centered at the nine vertices $0^{8}$, $\frac{1}{2}^{8},\left(\frac{1}{2}^{6}-\frac{1}{2}\right)-\frac{1}{2}$ of the Delaunay simplex, so $C M\left(E_{7}\right) \geq 9$. To obtain an upper bound we observe, as in the corresponding proof for $D_{3}$ (Theorem 8 ), that the covering ball centered at any lattice point is contained in the union of the Delaunay polytopes of which that point is a vertex. Points in a Delaunay orthoplex are therefore covered at most eight times, and points in a Delaunay simplex at most nine times. 
$E_{7}$ may be defined to consist of those vectors $x_{0} x_{1} \cdots x_{7} \in E_{8}$ for which $\sum x_{i}=0$, and has $R^{2}=\frac{3}{2}$. The dual lattice $E_{7}^{*}$ is generated by $E_{7}$ and the vector

$$
\left(\frac{3}{4}\right)^{2}\left(-\frac{1}{4}\right)^{6},
$$

and has $R^{2}=\frac{7}{8}$ (see [3, Chapter 4, Section 8.2], [4], [5], and [20]). The theta series of $E_{7}$ and $E_{7}^{*}$ are

$$
\begin{aligned}
& 1+126 q^{2}+756 q^{4}+2072 q^{6}+\cdots \\
& 1+56 q^{3 / 2}+126 q^{2}+576 q^{7 / 2}+\cdots
\end{aligned}
$$

respectively.

Theorem 11.

$$
C M\left(E_{7}\right)=28, \quad C M\left(E_{7}^{*}\right)=9 .
$$

Proof. (i) The Delaunay polytopes for $E_{7}$ are simplexes and Hesse polytopes $3_{21}$ (see [3]-[5]). To understand the Hesse polytope it is helpful to think of an icosahedron, whose 12 vertices lie in four layers of sizes $1+5+5+1$. The 56 vertices of the Hesse polytope similarly fall into four layers of sizes $1+27+$ $27+1$, as shown in Table 5 (see Table VIII of [4]). The Schläfli polytope $2_{21}$ is described below when we discuss $E_{6}$.

The vectors in Table 5 are in fact the minimal vectors of $E_{7}^{*}$. The pericenter of the first 28 vectors in the table is

$$
\frac{1}{4}-\frac{1}{12} 6 \frac{1}{4}
$$

and their perinorm is $\frac{4}{3}$, showing that $C M\left(E_{7}\right) \geq 28$. However, there are several inequivalent ways to choose a set of 28 overlapping balls. We conjecture that the above set has the smallest perinorm.

Table 5. Sections of the Hesse polytope $3_{21}$.

\begin{tabular}{lrl}
\hline \multicolumn{1}{c}{ Coordinates } & Number & \multicolumn{1}{c}{ Shape } \\
\hline$\frac{3}{4}-\frac{16}{4} \frac{3}{4}$ & 1 & Zenith \\
$\frac{3}{4}\left(\frac{3}{4}-\frac{1}{4}^{5}\right)-\frac{1}{4}$ & 6 & 27 vertices, \\
$\frac{1}{4}\left(-\frac{3^{2}}{4} \frac{1}{4}^{4}\right) \frac{1}{4}$ & 15 & forming a \\
$-\frac{1}{4}\left(\frac{3}{4}-\frac{15}{4}\right) \frac{3}{4}$ & 6 & Schläfli polytope \\
$\frac{1}{4}\left(-\frac{3}{4} \frac{1}{4}^{5}\right)-\frac{3}{4}$ & 6 & 27 vertices, \\
$-\frac{1}{4}\left(\frac{32}{4}-\frac{1}{4}\right)^{4}-\frac{1}{4}$ & 15 & forming a \\
$-\frac{3}{4}\left(-\frac{3}{4} \frac{1}{4}^{5}\right) \frac{1}{4}$ & 6 & Schläfli polytope \\
$-\frac{3}{4} \frac{16}{4}-\frac{3}{4}$ & 1 & Nadir \\
\hline
\end{tabular}


To prove $C M\left(E_{7}\right) \leq 28$ we follow our usual method. From the theta series (21) there are 2955 vectors of norm $<4 R^{2}=6$ to be considered. The fundamental simplex is defined by

$$
\begin{gathered}
x_{0} \leq x_{1}, \quad x_{1} \leq x_{2}, \quad \ldots, \quad x_{6} \leq x_{7}, \\
x_{4}+\cdots+x_{7}-x_{0}-\cdots-x_{3} \leq 1
\end{gathered}
$$

The exclusion graph has 42 nodes, and we must consider all maximal independent sets $\mathscr{S}$ of size $\geq 29$. There is a set $\left(\mathscr{S}_{1}\right.$ say) of 12 isolated nodes. It is easy to find by hand a set of 13 disjoint edges, and in four distinct ways. Hence there are at most $42-13=29$ nodes in $\mathscr{S}$. Furthermore, an independent set of size 29 must contain every node not involved in these 13 edges. In this way we find a set $\left(\mathscr{S}_{2}\right)$ of six further nodes that must belong to $\mathscr{S}$. Furthermore, the six nodes $\left(\mathscr{S}_{3}\right)$ joined to $\mathscr{S}_{2}$ are not in $\mathscr{S}$. This reduces the graph to $42-12-6-6=18$ nodes in which we must find all independent sets of size $29-12-6=11$. In the reduced graph there is a node $x$ of degree 8 which therefore cannot belong to $\mathscr{S}$, and a node $y$ of degree 6 . If $y \in \mathscr{S}$ there is a unique choice for $\mathscr{S}$, which has perinorm $\frac{14}{9}>\frac{3}{2}$; and if $y \notin \mathscr{S}$, then $|\mathscr{S}| \leq 28$.

(ii) The proof for $E_{7}^{*}$ is much easier. From (22) there are 759 vectors to be considered. The exclusion graph has 15 nodes and is shown in Fig. 3.

There is a unique maximal independent set, of size 9 , consisting of the points
(1) $-\frac{7}{4} \frac{17}{4}$,
(2) $-\frac{5}{4}-\frac{1}{4} \frac{3}{4}^{3}$,
(3) $-10^{4}\left(\begin{array}{ll}1 & 0^{2}\end{array}\right)$,
(4) $-\frac{3}{4}\left(-\frac{3}{4} \frac{1}{4}^{3}\right) \frac{1}{4}^{3}$

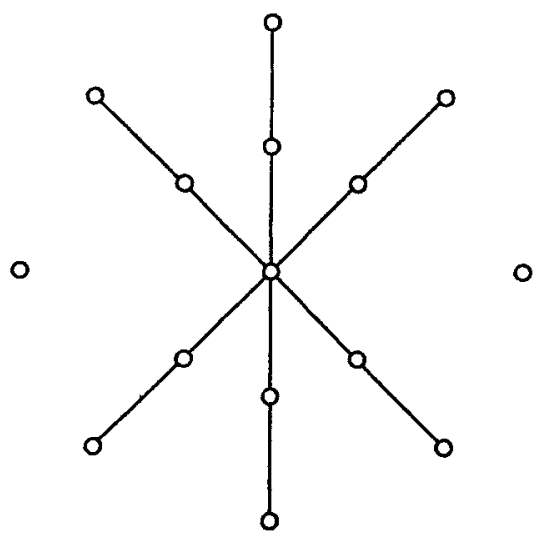

Fig. 3. Exclusion graph for $E_{7}^{*}$. 
(a)

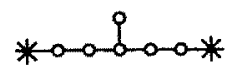

(c)

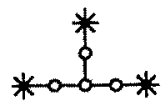

(b)

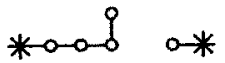

(d)

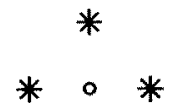

Fig. 4. (a) The $E_{7}^{*}$ lattice and (b) the polytope formed by the centers of nine intersecting balls. (c) The $E_{6}^{*}$ lattice and (d) the analogous 6-vertex polytope.

with pericenter

$$
-10^{4} \frac{1}{3}^{3}
$$

and periradius $\frac{5}{6}<\frac{7}{8}$. Thus $C M\left(E_{7}^{*}\right)=9$.

The proof also shows that the configuration of nine overlapping balls in $E_{7}^{*}$ is unique.

The points of $E_{7}^{*}$ fall into two orbits under the corresponding affine Weyl group, as indicated by the stars in Fig. 4(a), using the notation of [4]. The above nine points form two regular simplexes of dimensions 2 and 5 , as shown in Fig. 4(b), so their convex hull is the cross-join of these two simplexes.

$E_{6}$ may be defined to consist of those vectors $x_{0} x_{1} \cdots x_{7} \in E_{8}$ for which $x_{0}+x_{7}=x_{1}+\cdots+x_{6}=0$, and has $R^{2}=\frac{4}{3}$. The dual lattice $E_{6}^{*}$ is generated by $E_{6}$ and the vector

$$
0-\frac{22}{3} \frac{1}{3}^{4} 0
$$

and has $R^{2}=\frac{2}{3}$ (see [3, Chapter 4, Section 8.3], [4], [5], and [19]). The theta series of $E_{6}$ and $E_{6}^{*}$ are

$$
\begin{aligned}
& 1+72 q^{2}+270 q^{4}+\cdots, \\
& 1+54 q^{4 / 3}+72 q^{2}+\cdots,
\end{aligned}
$$

respectively.

\section{Theorem 12.}

$$
C M\left(E_{6}\right)=17, \quad C M\left(E_{6}^{*}\right)=6 .
$$

Proof. (i) The Delaunay polytopes for $E_{6}$ are Schläfli polytopes in two orientations [4]. The Schläfli polytope has 27 vertices, which may be taken to be the 
following:

$$
\begin{aligned}
& \text { (6) } \frac{1}{2}\left(\frac{5}{6}-\frac{15}{6}\right)-\frac{1}{2}, \\
& \text { (15) } 0\left(-\frac{22}{3} \frac{14}{3}\right) 0 \\
& \text { (6) }-\frac{1}{2}\left(-\frac{15}{6} \frac{5}{6}\right)-\frac{1}{2} .
\end{aligned}
$$

Each vertex has 16 neighbors, and the perinorm of such a set of 17 vectors is $\frac{5}{4}<\frac{4}{3}$, showing that $C M\left(E_{6}\right) \geq 17$. We will see that this is the only way a point can be covered 17 times.

To obtain an upper bound we consider the 343 vectors (from (24)) of norm $<4 R^{2}=\frac{16}{3}$. The fundamental simplex for $E_{6}$ may be defined by the inequalities

$$
\begin{array}{cl}
x_{1} \leq x_{2}, & x_{2} \leq x_{3}, \quad \ldots, \quad x_{5} \leq x_{6}, \\
x_{0}-x_{7} \leq 1, & x_{0}+\cdots+x_{3} \geq x_{4}+\cdots+x_{7} .
\end{array}
$$

However, this simplex is cut into two equal parts by the wall of one of the Schläfli polytopes, and so we may assume that a thickest point $P$ belongs to the semisimplex defined by (26) and the extra inequality

$$
2\left(x_{1}+x_{6}\right) \geq x_{2}+x_{3}+x_{4}+x_{5} .
$$

We now use AMPL and MINOS (see footnote 3) to show that just 20 of the 343 vectors are within $R$ of the semisimplex. A second application of AMPL and MINOS (see footnote 2) shows that the perinorms of all 18-subsets of this set of 20 are $\geq \frac{4}{3}$. Hence $C M\left(E_{7}\right) \leq 17$. Another calculation of the same type shows that there is a unique set of 17 out of the 20 with perinorm $<\frac{4}{3}$.

(ii) There is only one type of Delaunay polytope for $E_{6}^{*}$, which is the convex hull of three equilateral triangles in three orthogonal planes-we call this the cross-join of three triangles. It has nine vertices, for example

$$
\begin{aligned}
& \text { (3) } 0\left(-\frac{2}{3} \frac{1}{3} \frac{1}{3}\right) 00000 \text {, } \\
& \text { (3) } 0000\left(\frac{2}{3}-\frac{1}{3}-\frac{1}{3}\right) 0 \text {, } \\
& \text { (3) }\left\{\begin{array}{l}
\frac{1}{2} \frac{1}{6} \frac{1}{6} \frac{1}{6}-\frac{1}{6}-\frac{1}{6}-\frac{1}{6}-\frac{1}{2}, \\
-\frac{1}{2} \frac{1}{6} \frac{1}{6} \frac{1}{6}-\frac{1}{6}-\frac{1}{6}-\frac{1}{6} \frac{1}{2}, \\
0-\frac{1}{3}-\frac{1}{3}-\frac{1}{3} \frac{1}{3} \frac{1}{3} \frac{1}{3} 0,
\end{array}\right.
\end{aligned}
$$

whose center is the hole

$$
0 \frac{1}{3} \frac{1}{3} \frac{1}{3}-\frac{1}{3}-\frac{1}{3}-\frac{1}{3} 0
$$

The best we can do with these centers is to pick two out of each triple, so $C M\left(E_{6}^{*}\right) \geq 6$. For example, taking the first two of each triple in (28), we find that 
the pericenter is

$$
0000 \frac{1}{6} 000-\frac{1}{6} \quad 0
$$

and the perinorm is $\frac{11}{18}$. These six centers form what we call the separated join of three intervals, and Fig. 4(c) and (d) are analogous to Fig. 4(a) and (b).

To obtain an upper bound we consider the 127 vectors (from (25)) of norm $<4 R^{2}=\frac{8}{3}$. Of these just nine are within $R$ of the fundamental simplex (26). After a suitable translation of coordinates those nine coincide with (28).

\section{The Leech Lattice $\Lambda_{24}$}

For the definition and properties of this lattice see [3]. The covering radius $\boldsymbol{R}=\sqrt{2}$ and there are 307 distinct types of hole, namely 23 types of deep hole and 284 types of shallow hole [2], [3, Chapters 23 and 25].

\section{Theorem 13.}

$$
C M\left(\Lambda_{24}\right)=25
$$

We begin with a lemma.

Lemma 14. Let $h$ be a hole in $\Lambda_{24}$, let $\Pi$ be the Delaunay polytope containing $h$, and let $v_{1}$ be a vertex of $\Pi$. If the closed ball of radius $R$ around $h$ intersects the boundary of $\Pi$ at some point of a face $\pi$, then $v_{1}$ is a vertex of $\pi$.

Proof. Let the vertices of $\Pi$ be $v_{1}, \ldots, v_{M}$. As in Chapter 23, p. 481, of [3] we may choose a point $c$ (outside the space if $h$ is a shallow hole) so that $N\left(v_{i}-c\right)=$ $2,\left(v_{i}-c\right) \cdot\left(v_{j}-c\right)=0,-1$ or -2 , for $i, j=1, \ldots, M, i \neq j$. Any point $P$ on a face not containing $v_{1}$ can be written as

$$
P-c=\sum_{i=2}^{M} \lambda_{i}\left(v_{i}-c\right), \quad \lambda_{i} \geq 0, \quad \sum_{i=2}^{M} \lambda_{i}=1 .
$$

Then $N\left(P-v_{1}\right)=N(P-c)-2(P-c) \cdot\left(v_{1}-c\right)+N\left(v_{1}-c\right) \geq N(P-c)+2>2$

Lemma 14 implies that the ball of radius $R$ around $v_{1}$ is strictly inside the union of all Delaunay polytopes that have $v_{1}$ as a vertex.

Proof of Theorem 13. (i) Suppose $P$ is in a Delaunay polytope containing a shallow hole. All such polytopes are simplexes. By the lemma, $P$ is contained in at most the 25 balls centered at the vertices of the simplex. The shallow hole itself is contained in these 25 balls. The perinorm of these 25 vertices ranges from $2-\frac{2}{25}$ to $2-1 / 4900[3$, p. 521$]$. 
(ii) Suppose $P$ is in a Delaunay polytope containing a deep hole $h$. In this case the point $c$ used in proving the lemma coincides with $h$. Let $v_{1}, \ldots, v_{\mu} \in \Lambda_{24}$ be the vertices of a component of the hole diagram [3, p. 481]. Then $P$ is not in the interior of all $\mu$ balls of radius $R$ centered at $v_{1}, \ldots, v_{\mu}$. For if it were, then we would have $N\left(P-v_{i}\right)<2, i=1, \ldots, \mu$, hence

$$
N(P-h)<2(P-h) \cdot\left(v_{i}-h\right)
$$

However [3, p. 483], there are positive integers $m_{1}, \ldots, m_{\mu}$ such that

$$
\sum_{i=1}^{\mu} m_{i}\left(v_{i}-h\right)=0
$$

Therefore, multiplying (29) by $m_{i}$ and summing, we have

$$
\sum_{i=1}^{\mu} m_{i} N(P-h)<0
$$

which is impossible. It follows that the number of open balls containing $P$ is at most the number of vertices of the Delaunay polytope, minus the number of components of the hole diagram. This quantity is equal to 24 , independent of the type of deep hole [3, Table 23.1].

\section{Acknowledgments}

We are very grateful to Steve Fortune and David Gay for their assistance in using AMPL [9] and MINOS [15].

\section{References}

1. N. Bourbaki, Groupes et Algèbres de Lie, Chapitres 4, 5 et 6. Hermann, Paris, 1968.

2. J. H. Conway, R. A. Parker, and N. J. A. Sloane, The covering radius of the Leech lattice, Proc. Roy. Soc. London Ser. A, 380 (1982), 261 290. A revised version appears as Chapter 23 of [3].

3. J. H. Conway and N. J. A. Sloane, Sphere Packings, Lattices and Groups, Springer-Verlag, New York, 1988.

4. J. H. Conway and N. J. A. Sloane, The cell structures of lattices, in Miscellanea mathematica, ed. P. Hilton et al., Springer-Verlag, New York, 1991, pp. 71 107.

5. H. S. M. Coxeter, Regular Polytopes, 3rd edn., Dover, New York, 1973.

6. D. J. Elzinga and D. W. Hearn, Geometrical solutions for some minimax location problems, Transportation Sci., 6 (1972), 379-394.

7. D. J. Elzinga and D. W. Hearn, The minimum covering sphere problem, Management Sci., 19(1) (1972), 96-104.

8. D. J. Elzinga and D. W. Hearn, The minimum sphere covering a convex polyhedron, Naval Res. Logist. Quart., 21 (1974), 715-718.

9. R. Fourer, D. M. Gay, and B. W. Kernighan, AMPL: A modeling language for mathematical programming, Management Sci., 36 (1990), 519-554. 
10. J. E. Humphreys, Introduction to Lie Algebras and Representation Theory, Springer-Verlag, New York, 1972.

11. Mathlab Group, MACSYMA Reference Manual, Version 10, Laboratory for Computer Science, MIT, Cambridge, MA, 1983.

12. J. E. Mazo and A. M. Odlyzko, Lattice points in high-dimensional spheres, Monatsh. Math., 110 (1990), 47-61.

13. N. Megiddo and K. J. Supowit, On the complexity of some common geometric location problems, SIAM J. Comput., 13 (1984), 182-196.

14. B. A. Murtagh and M. A. Saunders, Large-scale linearly constrained optimization, Math. Programming, 14 (1978), 41-72.

15. B. A. Murtagh and M. A. Saunders, MINOS 5.1 User's Guide, Technical Report SOL 83-20R, Dept. Operations Research, Stanford University, Stanford, CA, Jan. 1987.

16. F. P. Preparata and M. I. Shamos, Computational Geometry: An Introduction, Springer-Verlag, New York, 1985.

17. W. D. Smith, Studies in Computational Geometry Motivated by Mesh Generation, Ph.D. Dissertation, Program in Applied and Computational Mathematics, Princeton University, Sept. 1988.

18. J. M. Sullivan, A Crystalline Approximation Theorem for Hypersurfaces, Ph.D. Dissertation, Department of Mathematics, Princeton University, 1990.

19. R. T. Worley, The Voronoi region of $E_{6}^{*}, J$. Austral. Math. Soc. Ser. A, 48 (1987), 268-278.

20. R. T. Worley, The Voronoi region of E*, SIAM J. Discrete Math., 1 (1988), 134-141.

Received October 3,1990, and in revised form March 14, 1991. 